\title{
Ultrasonography of the biliary tract - up to date. The importance of correlation between imaging methods and patients' signs and symptoms.
}

\author{
Radu Badea ${ }^{1}$, Răzvan Zaro², Marcel Tanțău², Liliana Chiorean ${ }^{3}$
}

${ }^{1}$ Ultrasound Imaging Laboratory, Department of Imaging and Radiology, ${ }^{2}$ Gastroenterology Department, $3{ }^{\text {rd }}$ Medical Clinic, „Octavian Fodor” Regional Institute of Gastroenterology and Hepatology, „Iuliu Hatieganu” University of Medicine and Pharmacy, Cluj-Napoca, Romania, ${ }^{3}$ Med. Klinik 2, Caritas Krankenhaus Bad Mergentheim, Germany

\begin{abstract}
Ultrasonography is generally accepted and performed as a first choice imaging technique in patients with jaundice. The method allows the discrimination between cholestatic and mechanical jaundice. The existing procedures are multiple: gray scale, Doppler, i.v. contrast enhancement, elastography, tridimensional ultrasonography, each of these with different contribution to the positive and differential diagnosis regarding the nature of the jaundice. The final diagnosis is a multimodal one and the efficiency is dependent on the level of the available technology, the examiner's experience, the degree and modality of integration of the data within the clinical context, as well as on the portfolio of available imaging procedures. This review shows the main ultrasonographic methods consecrated in the evaluation of the biliary tree. It also underlines the integrated character of the procedures, as well as the necessity to correlate with other imaging methods and the clinical situation.

Keywords: biliary tree, contrast enhanced ultrasound, elastography, echoendoscopy
\end{abstract}

\section{Introduction}

Biliary tract conditions are classified into congenital (stenosis/atresia, choledocal cysts) and acquired (inflammatory: acute cholangitis, primary sclerosing cholangitis - PSC or tumoral). The diagnosis is based on various and complex imaging procedures: ultrasonography (US), followed by computed tomography (CT), magnetic resonance imaging (MRI), magnetic resonance cholangiopancreatography (MRCP), and endoscopic retrograde pancreatography (ERCP). Their results have to be correlated with the clinical presentation.

US represents the first choice imaging method used after the clinical exam, being useful in demonstrating biliary obstruction (identifing intra and extrahepatic biliary

Received 30.05.2015 Accepted 29.06.2015

Med Ultrason

2015, Vol. 17, No 3, 383-391

Corresponding author: Răzvan Zaro

$3^{\text {rd }}$ Medical Clinic,

19-21 Croitorilor Street,

400162, Cluj Napoca, România

E-mail: Razvan.Zaro@umfcluj.ro tract dilatation), communication with the digestive tract (aerobilia), tumors, or associated pathology. US is highly sensitive in the diagnosis of lithiasis (accuracy is higher when the calculi are intrahepatic) [1,2]. It is currently used for guiding interventional procedures and for indicating the position and functionality of the drainage tubes (placed either during endoscopy or surgery. Combining several US procedures, the accuracy of US examination can be increased. The Doppler technique reveals arterial and venous circulatory anomalies and vessel dislocation. The tridimensional technique (3D) brings topographic information [3]. Contrast enhanced ultrasonography (CEUS) shows the circulatory alterations of the small vessels. Elastography allows a characterization of the tissue stiffness. High-resolution endoscopic US, performed with microprobes, visualizes very small tumors or calculi.

The purpose of this review is to highlight the current accomplishments of US, due to the added value brought by CEUS, elastography, and 3D US, as well as the alternative use of other ultrasonographic techniques. It also includes the epidemiology and a brief presentation of the clinical features of the biliary tract conditions. 


\section{Ultrasonographic techniques and procedures}

Grey scale US: represents the basic examination technique. It allows the identification of organic changes and biliary tract dilations, having a good temporal and spatial resolution. It is an operator-dependent technique.

I.V. contrast enhanced US (CEUS). This is a vascular (angiospecific) investigation which provides information about the circulatory bed. The first good practice guidelines were publised by the European Federation of Ultrasonography in Medicine and Biology (EFSUMB) in 2004 [4], being updated in 2008 [5] and in 2012, with the participation of the World Federation of Ultrasonography (WFUMB) [6,7]. In 2012 recommendations on the applicability of CEUS in non-hepatic pathology were also added [8]. In order to sustain and illustrate the guidelines, commentaries on this subject were elaborated and published $[9,10]$. In general, the role of CEUS is oriented towards the definition and characterization of tumors, mainly liver tumors, monitorization of systemic therapy, surgery, or percutaneous ablation efficiency, vessels characterization, or posttransplatation follow up. The data regarding tumor or inflammatory pathology of the intra or extrahepatic biliary ducts is relatively reduced. The CEUS examination consists of real-time evaluation of the enhancement/washout vascular ,pattern” of the contrast agent (CA) in the vascular bed; by this, it allows the distinction between normal and pathological, inflammatory and tumoral. It also allows the identification of all vascular „phases": arterial, portal, and delayed/late venous (tissue) phase. The region of interest (ROI) must be observed for 3 to 5 minutes and comparatively evaluated with a normal, refference tissue. The procedure is based on the generation of harmonic echoes by microbubbles exposed to low acoustic power sound waves. The CA that are used today have no cardiac or renal toxicity. The gas microbubbles have a small diameter (a mean of $5-8$ microns), are i.v. administered and are kept strictly intravascular. The CA can be introduced alse into the bile ducts, either by injection during percutaneous drainage procedures or by a adminstration through drainage tubes, in this situation having an ultrasonographic ,fistulography” character [11]. CEUS may be combined with endoscopic ultrasonography (CE CEUS), using a high MI or low MI, the latter being similar to transabdominal CEUS $[12,13]$. Combination with spectral Doppler analysis, can detect the presence of arterioles within pancreatic ducts adenocarcinomas, an aspect that allows a better angiographic perfusion analysis [14].

Intraductal endoscopic US. It consists of using elastic high frequency (12-30 MHz) probes attached on the guiding wire, probes introduced through the operative channel of the endoscope and then through the papilla inside the main bile duct [15]. The round image, resulted from the continuous rotation of the transducer, allows the visualisation of the ductal wall stratification at a resolution close to that of the microscope. The image is in grey scale, but it can be combined with CEUS.

Intraoperative CEUS exploration (IO-CEUS). It is often used in patients with hepatocarcinoma (HCC) or resecable liver metastases. Current studies have shown superior sensitivity and specificity of IO-CEUS compared with MRI and $\mathrm{CT}$, in predicting tumor resecability $[16,17]$. IO-CEUS is useful in characterizing new HCC nodules in patients with cirrhosis, nodules detected during intraoperative ultrasound (IOUS) [18]. IO-CEUS may be used in conducting combined therapy, surgery, and ablation, of the HCC. Applications of this method in the pathology of the biliary tract are under development.

Elastography. This is a procedure able to represent and quantify (in meters/second or kiloPascals), the stiffness of the examined tissue. There are several elastographic techniques [19]. In the last years it has become a standard in the noninvasive evaluation of liver stiffness which directly correlates with the degree of fibrosis [20]. In the case of the biliary tract, a transabdominal approach may be attempted if the masses are large. Elastography can be combined with endoscopic US in case of small ampullary/periampullary tumors [21]. EFSUMB and WFUMB have published guideliness and recommendations for the clinical applicability of elastography [19].

Tridimensional (3D) US. 3D US is a procedure consisting of simultaneous representation of perpendicular ultrasonographic views, automatically generated by special transducers. Modern equipment allows a digital „fill in” of the spaces between the planes in a manner that allows the generation of volumetric images. The 3D exploration presents a spatial representation of the organs, allows their rotation for a better identification of certain details and facilitates the substraction of the structures with different echogenicity. The method is a sophisticated procedure with disadvantages related to the complexity of the equipment and dependence on the operator's experience. Obstetrical applications are the most well-known, but there are publications that show the use of $3 \mathrm{D}$ in characterizing and quantifing liver, breast, and thyroid tumors [22] In the case of the biliary tract, 3D US allows the „extraction” of the biliary ducts from the liver paranchyma by using special software (mainly the „transparency" mode) for a better representation, especially when they are dilated [3].

\section{Normal aspect}

The interpretation of the normal US aspect of the biliary tract is suggested by a few simple rules: a) right and 
left biliary ducts are the only intrahepatic biliary tract visibile; b) the main bile duct has a calibre of 6-8 $\mathrm{mm}(9$ $\mathrm{mm}$ acceptable if the patient had a cholecistectomy); c) the ampulla of Vater is not visible; d) the Wirsung duct is only visible on short segments and has a calibre under 2 $\mathrm{mm}$; e) the content of the biliary tract in transsonic. The CEUS examination, elastography and 3D are not relevant in the characterization of the normal aspect, with the exception of the gallbladder.

\section{Pathology}

Biliary tract lithiasis. About $15 \%$ of the patients with intra and extrahepatic lithiasis develop choledochal calculi as well [23]. The risk factors for choledochal lithiasis are the same as those for biliary lithiasis [24]. Genetic and ethnic predisposition determines an increase of lithogenic susceptibility. The typical US appearance is that of an echoic image with acoustic shadowing. The accuracy of US is good when it comes to intrahepatic lithiasis, but it is significantly lower in cases of lower, subpapilary locations. A 3D examination can identify an „amputation” of the choledoc, without defining the nature of the obstacle (fig 1). Elastography and CEUS have no indication in the diagnosis of this condition.

Acute angiocholitis. This is a syndrome generated by the combination between biliary obstruction and pathological bacterial colonization. The main causes are: lithiasis $(28-70 \%)$, benign stenosis $(5-28 \%)$, malignant tumors (10-57\%) and obstruction of endoscopic stents $(18 \%)$ [25]. The diagnosis of angiocholitis is initially based on the Charcot triad and biological data. Grey scale US shows dilatations of the biliary tree and brings information about the level and nature of the obstacle. Rarely, US may also reveal thickening of the choledochal mucosa, suggesting edema. In $10-20 \%$ of the cases small calculi may be missed when they are located close to the papilla [2]. Biliary duct abscesses within the liver parenchyma may develop as a result of unproperly treated

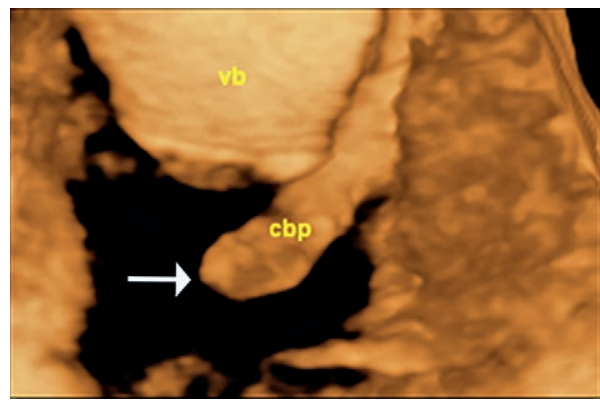

Fig 1. Main bile duct lithiasis (3D aspect); vbbiliary vesicle, $\mathrm{cbp}$ - main bile duct angiocholitis episodes. CEUS strengthens the diagnosis by revealing focal unenhacing areas during the arterial phase. The surrounding liver parenchyma is hyperenhancing, an expresion of the congestion surrounding the abscesses (fig 2) [26]. 3D US can accurately reveal the dilatation of the intrahepatic bile ducts (fig 3). MRCP and endoscopic US are reliable in detecting edema of the choledochal wall and identifing the obstacle. Endoscopic US has a high rate of detection of gallbladder and choledochal lithiasis (sensitivity $91-00 \%$, specificity $96-99 \%$ ), similar to MRCP [27]. Abdominal CT is superior to US in establishing the site of the obstruction [28], biliary dilatation being registered in $82 \%$ of the patients [29]. ERCP represents the basic procedure, being both diagnostic and therapeutic. During the biliary tract exploration, disobstruction is also performed, with a rate of calculus extraction between $90-95 \%$ [30].

Primary sclerosing cholangitis (PSC) is a chronic, progressive disease, which has an autoimune component; it is characterized by inflammation, fibrosis, and intra and extrahepatic ducts stenosis. There is an increase of the cholestasis enzymes and a biliary retention, while the autoimunity marker p-ANCA is positive. US shows

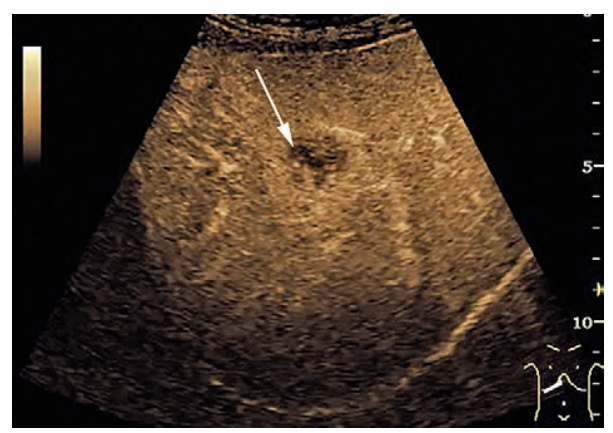

Fig 2. CEUS in a case of angiocholitis. An abscess is detected in arterial phase (arrow).

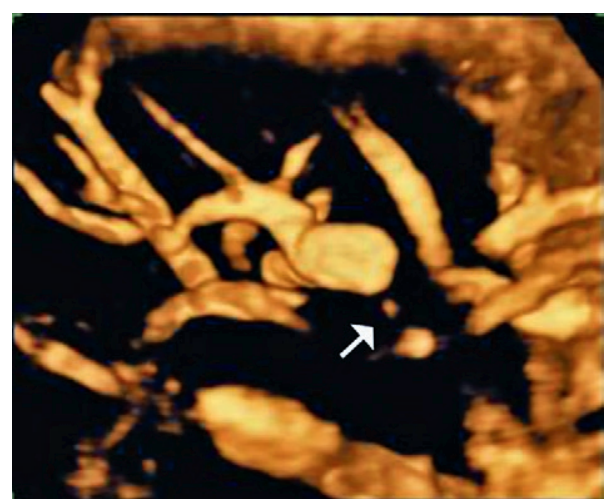

Fig 3. Dilated biliary tree (3D aspect). Cause of the cholestasis is represented by a tumor (arrow). 
thickening of the ductal wall and segmentary dilations [31]. CEUS is useful in the differential with biliary tract tumors, in cases where there is important hypertrophy of the ductal wall. The CEUS appearance is that of enhancement of the ROI during the arterial phase and lack of washout during the venous phase. The 3D US exploration is not relevant in this case. Elastography of the liver may reveal increased stiffness when there is fibrosis, but it does not provide information about the biliary pathology. CT shows biliary ducts inflammation and saccular dilatations of the biliary tree. The final diagnosis is established based on the MRCP and ERCP which show multiple strictures accompanied by normal or dilated intra and extrahepatic biliary ducts. Due to the noninvasive character and the comparable results of the MRCP with ERCP, MRCP is the preferred diagnosis method [32]. ERCP is reserved for patients who cannot undergo MRCP or those who need endoscopic therapy.

Benign tumors of the biliary tract. These are rare conditions discovered either by chance or when they produce clinically manifested biliary obstruction. Adenomas are isoechoic masses, related to the liver parenchyma, homogeneous or heterogeneous (calcifications), which associate dilatations of the suprajacent biliary ducts. CEUS exploration reveals enhancement during the arterial phase and no washout during the venous phase. Cystadenomas are usually solitary tumors that have a malignant degeneration potential. They have a cystic aspect, with dilatation of the biliary tract (fig 4) [33]. The differential diagnosis is made with hydatid cysts, metastases, and abscesses. The 3D examination shows the cystic mass and establishes the location and anatomic relationships with the surrounding structures (fig 5). Elastography is irrelevant for the diagnosis. Intraductal US reveals the lack of extension beyond the biliary duct walls, which consolidates the benign diagnosis.

Benign tumors of the ampulla of Vater: represent about $10 \%$ of the ampullary tumors, adenomas being the most frequent (villous, tubulovillous) [34]. The symptoms may vary between cholestatic extrahepatic jaundice, acute pancreatitis, and upper digestive tract hemorrhage. Conventional US and CT show dilatations of the biliary tract and, sometimes, depending on the size, even the tumor. When the tumor is visible on grey scale, CEUS shows homogeneous uptake during the arterial phase, while during the venous phase it becomes hypoenhancing [35]. 3D US brings useful topographic details. ERCP always detects the tumor [36] and additionally allows the placement of prosthetic devices in the choledoc, prelevation of biopsies and endoscopic resection [37]. The role of endoscopic US is represented by the preoperative evaluation and staging of ampullary carcinomas.

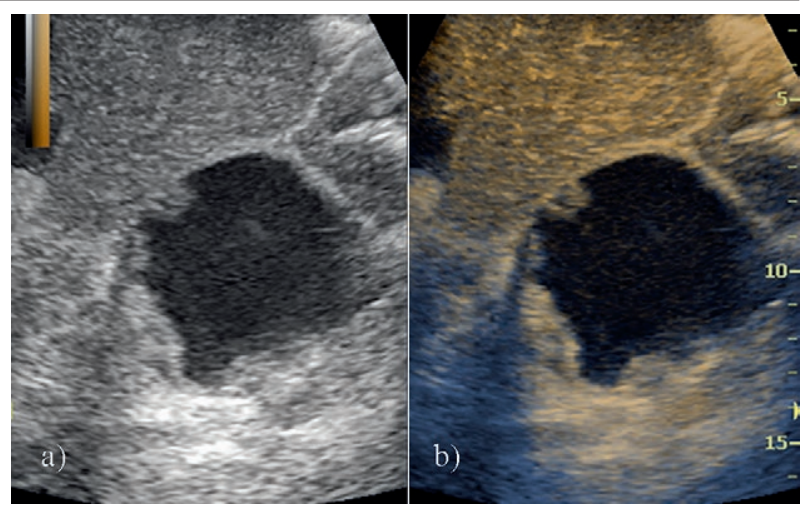

Fig 4. Cystadenoma of the biliary tree, a) grey scale ultrasound and b) CEUS evaluation

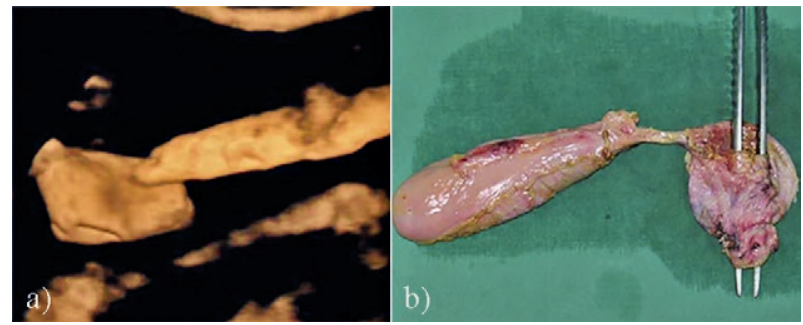

Fig 5. Choledocal cyst; a) 3D reconstructed ultrasonography; b) surgical specimen

A more confident discrimination between malignant and benign tumors may be realized by using elastography. Other examinations that may be used are represented by miniprobe intraductal US and narrow band magnification endoscopy buttheir role in the diagnosis being uncertain at the moment [38].

Cholangiocarcinoma (CCC): a primary, intra or extrahepatic (about 50-60\% of the cases), malignant tumor of epithelial origin. It represents $10-15 \%$ of all hepatobiliary cancers [39]. The mortality rate is high due to the advanced stage at the moment of the diagnosis [40]. The most significant symptom is represented by jaundice. Biology parameters show cholestasis, biliary retention, and increased tumor markers (CA 19-9 and CEA). The role of imaging is to diagnose CCC and to identify and establish the characteristics of the tumor, to detect possible secondary liver, nodal or peritoneal masses, and to define local contiguity and relationship to the liver pedicle, hepatic vein, biliary tract and inferior vena cava.

a) Intrahepatic CCC: this refers to a tumor found in the liver parenchyma, often in the periphery, subcapsular. US detects the dilatation of the suprajacent bile ducts and may show a solid, hyperechoic, homogeneous, or heterogeneous mass. The differential diagnosis with hepatocarcinoma (HCC) or liver metastases is difficult to establish (fig 7) [41]. Proving the invasion of the bile 
ducts consolidates the diagnosis of CCC. The CEUS aspect correlates with the size of the tumor and the proportion of fibrotic and necrotic tissue. In tumors smaller than $3 \mathrm{~cm}$, where the quantity of fibrotic tissue is higher and vascularity is unfluctuating, the CEUS aspect is homogeneous in the arterial phase, making the differentiation from HCC difficult. In the portal and delayed phases CCC present a more intense and quick washout of the $\mathrm{CA}$ than $\mathrm{HCC}$ [42]. As the mass increases in size, circulatory alterations will develop inside the tumor. There are four enhancement patterns in the arterial phase: irregular, circular hyperenhancement in the periphery (about 50\% of the cases); heterogeneous hyperenhancement; heterogeneous hypoenhancement, and homogeneous hypoenhancement [43]. Cystadenocarcinomas present hyperenhancement of the wall, septum, and parenchymal portion of the mass and hypoenhacement in the central area during the arterial phase; in the portal and delayed phases there is washout [44]. The CEUS aspect of intrahepatic CCC is similar to that of the metastases, independent of the arterial uptake, with the distinction that in the delayed phase there is intense washout [45]. 3DS examination visualizes the tumor without having additional diagnosis value. Elastography usually shows nonhomogeneous increased of the tumor stiffness. CT an MRI identify hyperenhancement in the delayed phase due to the diffusion of the contrast agent within the tumor interstitium. Chen et al found accuracy for intrahepatic CCC of $80 \%$ for CEUS and $67.5 \%$ for CT [46]. CT cholangiography has a diagnosis accuracy of up $94 \%$ for intrahepatic CCC [47]. Another constant element is the retraction of the capsule (in approximately $20 \%$ of the patients) due to the fibrotic nature of the tumor [48]. MRI images reveal various degrees of signal, in relationship with the level of fibrosis, necrosis, and quantity of mucin found in the tumor. In T1 intrahepatic CCC is hypo-/isointense, while in T2 it is hyperintense. After gadolinium administration there is minimal, progressive enhancement, especially in the periphery of the tumor, while in the delayed phases there is central enhancement [49]. In situations where the uptake pattern is similar with that of the HCC - some mixed types like cholagiocellular carcinoma, liver biopsy becomes mandatory. The specificity of positron emission computed tomography (PET - CT) in detecting small size intrahepatic CCC (down to $1 \mathrm{~cm}$ ) was reported to be around $85-95 \%$, one of the limitations being related to the impossibility of differentiating active inflammatory lesions from malignant ones [50].

b) Periductal cholangiocarcinoma (Klatskin tumor). This refers to tumors arising in the common bile duct or the proximal suprajacent biliary ducts. The differential diagnosis includes lymphoma, lymphosarcoma, ex- trinsec compressions or benign strictures. Grey scale US mainly shows symmetric dilatation of the bile ducts. The primary tumor is difficult to visualize, as it is usually a small size and isoechoic. On CEUS, a Klatskin tumor may show various enhancement patterns: arterial phase- hyper enhancing $(43.8 \%)$, isoenhancing $(43.8 \%)$, or hypoenhancing (12.4\%); during the portal venous and delayed phases the tumor is hypoenhancing in $93.8 \%$ of the cases [51]. Uptake distribution during the arterial phase may be hyperenhancing in the periphery ("rim-like"), uniform and homogeneous, or nonhomogeneous in $9.4 \%, 34.4 \%$ and $56.2 \%$ of the cases [51]. A comparative analysis of CEUS and contrast CT shows that the arterial phase uptake pattern and efficiency in detecting portal infiltration are similar. The sensitivity of CEUS for a Klatskin tumor is $93.8 \%$, superior to the sensitivity of contrast (fig 6) CT, which is $78.1 \%$ [51]. 3D US may be useful for a more accurate identification of the site of the biliary obstacle. Measuring the distance between the intrahepatic biliary blunt ends represents an auxiliary method of determining the tumor size. Elastography is useful when the tumor is large. In such circumstances the tumor is stiff in the vast majority of the cases, the procedure being useful in differentiating pathologic from normal liver tissue. CT is a non-invasive diagnosis method used to appreciate the level of obstruction and the local and distant extension. It has accuracy between 78 and $92 \%$ [52]. CT cholangiography is superior to conventional $\mathrm{CT}$, having sensitivity close to that of MRCP or ERCP [53]. Endoscopic US with fine needle aspiration (FNA) has accuracy up to $83 \%$ even without brush cytology [54]. The method of choice for biliary tract mapping is MRCP, being considered the optimal evaluation for patients with CCC. Accuracy in evaluating intraductal tumor extension varies between $71-96 \%$, and that of local lymph node extension is approximately $66 \%$ [55]. Among the invasive techniques ERCP and percutaneous transhepatic cholangiography must be noted. The diagnosis sensitivity of ERCP is 75\% (accuracy 95\%) [56]. ERCP allows the prelevation of cytology material through the brushing technique. Endoscopic cholangioscopy and intraductal ultrasonography are used on a smaller scale.

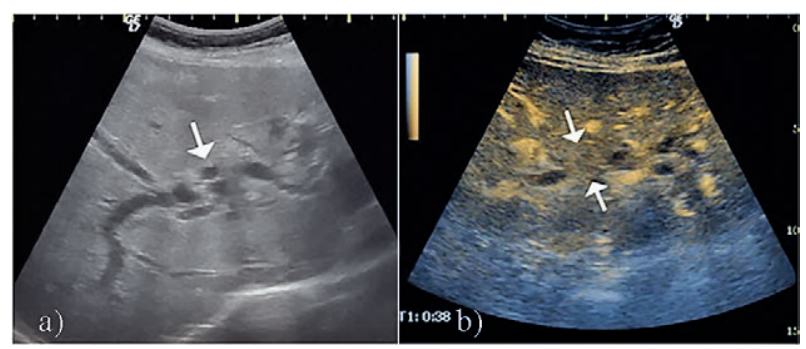

Fig 6. Intrahepatic cholangiocarcinoma (Klatskin tumor); a) 2D ultrasonography (arrow); b CEUS evaluation (arrows). 
c) Distal cholangiocarcinoma. It refers to inferior, extrahepatic tumors, anywhere distal to the cystic duct or ampulla of Vater. The symptoms include jaundice without pain, associating or not angiocholitis. US identifies the dilatation of the bile ducts and the marked dilatation of the gallbladder as well as the tumor itself. The tumor realizes a concentric, fusiform stenosis of the choledochal duct, has a parenchymal aspect and imprecise delineation. The fact that the Wirsung duct is not dilated orientates the diagnosis towards the biliary origin of the tumor. CEUS exploration increases the performance of US by differentiating CCC from benign lesions. During the arterial phase the appearance is not characteristic, it can be either hyper, hypo or isoenhancing, but in the delayed phase most of them are hypoenhancing [57]. 3D US brings useful information regarding tumor topography. Elastography demonstrates increased tumor stiffness only when the size of the tumor is large enough. Microprobe endoscopic exploration brings additional information by visualizing the tumor and the degree of invasion in the choledochal wall [58]. ERCP is highly predictive in the detection of extrahepatic CCC, allowing also the palliative therapy of the extrahepatic cholestatic jaundice by the placement of biliary prosthetic devices. CT and MRCP visualize the dilatation of the suprajacent bile ducts and the stenosis of choledocal segment affected by the tumor. Endoscopic US FNA has a role in the preoperative evaluation and in establishing resectability [59].

Ampullary carcinoma. This refers to an epithelial tumor arising from the ampulla of Vater. US can detect the dilatation of both the choledochal and the pancreatic ducts (in $75 \%$ and $67 \%$ of the cases) [60]. The CEUS aspect seems to be dependent of the histopathological type. Therefore, the papillary carcinoma has an oval shape enhancement of the CA, while the mixed type has an irregular uptake. 3D exploration may individualize the ampullary tumor. CT demonstrates local lymph node adenopathies and distant metastases, but lacks the spatial resolution to detect small size ampullary tumors protruding into the duodenum lumen. Choledochal and/ or pancreatic duct dilatation, accompanied by a sudden stop at the level of the ampulla (in the absence of other pathology) is suggestive of ampullary carcinoma (fig 7) [61]. ERCP allows the prelevation of biopsies and the decompression of the biliary tree by stenting, without being able to appreciate the extension of the tumor. MRI cholangiography may be used in diagnosis purposes in patients who cannot undergo ERCP. The MRI aspect is that of a mass with hypo T2 signal, situated at the level of the pancreatico-biliary junction. PTC may be used in patients where ERCP failed, the technique allowing cholangiography, brushing cytology (in cases where the tumor

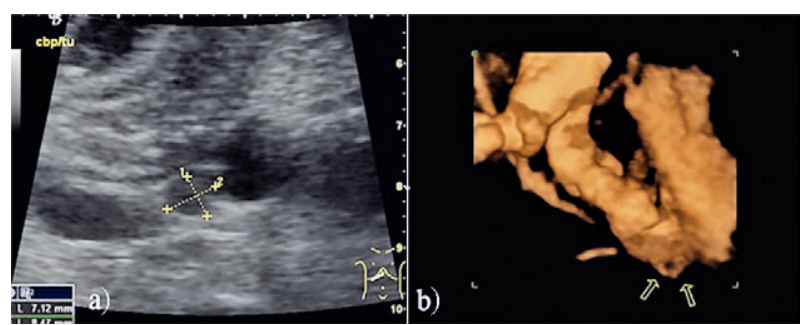

Fig 7. Ampulary carcinoma: a) 2D ultrasonography; b) 3D ultrasonography

is extended in the choledochal duct), and percutaneous biliary drainage. It cannot make any assumptions regarding the tumor extension into the duodenum or the pancreas. Endoscopic US is similar as sensitivity with ERCP in tumor detection, being superior to transabdominal US or $\mathrm{CT}$. Defrain et al showed a diagnosis accuracy of endoscopic US-FNA in evaluating ampullary tumors of $89 \%$ (sensitivity $82 \%$ and specificity $100 \%$ ), the method being useful especially in the preoperative evaluation (the accuracy of the T stage being 70-90\%) [62]. Endoscopic intraductal US is even more accurate in the evaluation of the T stage comparing with EUS [63].

Intrabiliary metastases. Their etiology is variable: digestive tract, pancreas, malignant melanoma, and less often, lung, breast, or genito-urinary tract. The liver metastases may determine extrinsec or intrinsec compression upon the intra or extrahepatic biliary ducts [64]. The US aspect is that of a circumscribed mass, within the biliary duct lumen, associate with suprajacent dilatation of the biliary tract. The image is not characteristic. CEUS exploration reveals arterial nonhomogeneous uptake during the arterial phase and venous washout. Elastography is irrelevant for the diagnosis. 3D US shows biliary tract "amputation" at the level of the obstruction, without bringing any additional information.

Portal cholangiopathy (PC). It develops in $80 \%$ of the patients with non-tumoral portal thrombosis [65]. PC was classified into three stage based on MRCP: stage I - minimal ductal irregularities; stage II - ductal stenosis without dilations; stage III (symptomatic) - ductal stenosis with dilations [65]. The condition is frequently detected $(81-100 \%)$ when there is extrahepatic portal obstruction [66]. Pathogenesis is represented by the extrinsic compression due to venous collaterals and biliary tree ischemia determined by the portal thrombosis. The US aspect is represented by thickening of the biliary walls and presence of a portal vascular signal on the Doppler exploration concomitant with portal hypertension signs. CEUS shows CA enhancement during the portal phase. Gallbladder varicose veins can be identified by greyscale and Doppler US, endoscopic US, and CEUS [67]. 
The main diagnostic imaging techniques are represented by MRI cholangio-portography and ERCP (which has the benefit of the endoscopic treatment).

Hemobilia. This is one of the rare causes of upper digestive tract hemorrhage, its origin being the intra or extrahepatic biliary tract. Among the most frequent causes are: abdominal trauma affecting the biliary tree, iatrogenic lesions (liver biopsy, hepatectomy, internal or external biliary drainage, invasive endoscopic procedures), choledochal calculi, alithiasic cholangitis, vascular pathology (portal hypertensive cholangiopathy, hepatic artery aneurism or vasculitis) and primary or secondary neoplastic pathology $[68,69]$. The most frequently encountered etiology is represented by hemobilia secondary to iatrogenic lesions, Green et al identifying it in $66.21 \%$ of the cases [70]. Grey scale US detects moderate dilatation of the biliary tree and low intensity echoes in bile duct lumen. CEUS exploration reveals the blood clots. Elastography and 3D US are irrelevant in this condition. MRI is superior in detecting the hemorrhage and identyfing the cause.

\section{Limitations of US}

Due to resolution issues, the smallest detectable lesions vary between 3 and $5 \mathrm{~mm}$ [71]. Subdiaphragmatic lesions may be missed on grey scale or CEUS, especially if steatosis is associated. CEUS exploration does not detect vascularization in normal circumstances. The spatial resolution of the method is not sufficient to visualize the slow circulation at this level. Elastography is nonspecific as it strictly differentiates stiff from soft tumors. 3D US is operator and equipment dependent and is not integrated with routine medical practice.

\section{Conclusions}

Biliary tract US is the first choice imaging method performed in patients with jaundice. Grey scale US remains the basic exploration technique, while there are several other auxiliary procedures: Doppler, CEUS, elastography, 3D US which bring highly valuable additional information in defining the type of jaundice, the site and nature of the obstacle. The combination of US with endoscopic techniques (elastography, FNA, ductal endoscopic US) significantly increases the value of the informations, but they also take US towards the invasive diagnosis. The method remains operator dependent and it is limited when there is superimposing gas or significant attenuation. The association with the clinical criteria is extremely important. The final diagnosis is realized by associating US with other biliary tract diagnosis proce- dures, their selection being decided after corroborating the US with the clinical and biological data.

Conflict of interests: none

\section{References}

1. Cooperberg PL, Schescke GA, Mathieson JR. The gallbladder and bile ducts. In: Goldberg BB, Petterson H (eds). The NICER Year Book 1996: Ultrasonography. Oslo, The NICER Institute 1996.

2. Nakayama F, Koga A. Hepatolithiasis: present status. World J Surg 1984; 8: 9-14.

3. Socaciu M, Badea R, Tanţău M, Iancu C, Tripon S, Caraiani $\mathrm{C}$. The performance of three-dimensional ultrasound reconstruction of the bile ducts in the diagnosis and assessment of extrahepatic cholestasis - a clinical trial on 48 patients. Med Ultrason 2008; 10: 21-28.

4. Albrecht T, Blomley M, Bolondi L, et al. Guidelines for the use of contrast agents in ultrasound. January 2004. Ultraschall Med 2004; 25: 249-256.

5. Claudon M, Cosgrove D, Albrecht T, et al. Guidelines and good clinical practice recommendations for contrast enhanced ultrasound (CEUS) - update 2008. Ultraschall Med 2008; 29: 28-44.

6. Claudon M, Dietrich CF, Choi BI, et al. Guidelines and good clinical practice recommendations for Contrast Enhanced Ultrasound (CEUS) in the liver - update 2012: A WFUMB-EFSUMB initiative in cooperation with representatives of AFSUMB, AIUM, ASUM, FLAUS and ICUS. Ultrasound Med Biol 2013; 39: 187-210.

7. Claudon M, Dietrich CF, Choi BI, et al. Guidelines and good clinical practice recommendations for contrast enhanced ultrasound (CEUS) in the liver--update 2012: a WFUMBEFSUMB initiative in cooperation with representatives of AFSUMB, AIUM, ASUM, FLAUS and ICUS. Ultraschall Med 2013; 34: 11-29.

8. Piscaglia F, Nolsoe C, Dietrich CF, et al. The EFSUMB Guidelines and Recommendations on the Clinical Practice of Contrast Enhanced Ultrasound (CEUS): update 2011 on non-hepatic applications. Ultraschall Med 2012; 33: 33-59.

9. Dietrich CF, Cui XW, Schreiber-Dietrich DG, Ignee A. EFSUMB guidelines 2011: comments and illustrations. Ultraschall Med 2012; 33 Suppl 1: S11-S21.

10. Dietrich CF, Schreiber-Dietrich D, Hocke M. Comments on the EFSUMB non-liver Guidelines 2011. Praxis (Bern 1994) 2012; 101: 1175-1181.

11. Ignee A, Baum U, Schuessler G, Dietrich CF. Contrastenhanced ultrasound guided percutaneous cholangiography and cholangiodrainage (CEUS-PTCD). Endoscopy 2009; 41: 725-726.

12. Dietrich CF, Ignee A, Frey H. Contrast-enhanced endoscopic ultrasound with low mechanical index: a new technique. Z Gastroenterol 2005; 43: 1219-1223. 
13. Kitano M, Sakamoto H, Matsui U, et al. A novel perfusion imaging technique of the pancreas: contrast-enhanced harmonic EUS. Gastrointest Endosc 2008; 67: 141-150.

14. Hocke M, Schulze E, Gottschalk P, Topalidis T, Dietrich CF. Contrast-enhanced endoscopic ultrasound in discrimination between focal pancreatitis and pancreatic cancer. World J Gastroenterol 2006; 12: 246-250.

15. Fujita N, Noda Y, Kobayashi G, et al. Intraductal ultrasonography (IDUS) for the diagnosis of biliopancreatic diseases. Intraductal ultrasonography (IDUS) for the diagnosis of biliopancreatic diseases. Best Pract Res Clin Gastroenterol 2009; 23: 729-742.

16. Fioole B, de Haas RJ, Wicherts DA, et al. Additional value of contrast enhanced intraoperative ultrasound for colorectal liver metastases. Eur J Radiol 2008; 67: 169-176.

17. Lu Q, Luo Y, Yuan CX, et al. Value of contrast enhanced intraoperative ultrasound for cirrhotic patients with hepatocellular carcinoma: a report of 20 cases. World J Gastroenterol 2008; 14: 4005-4010.

18. Torzilli G, Del Fabbro D, Palmisano A, Donadon M, Montorsi M. Contrast-enhanced intraoperative ultrasonography: a valuable and not any more monocentric diagnostic technique performed in different ways. Ann Surg 2007; 245: 152-153.

19. Bamber J, Cosgrove D, Dietrich CF, et al. EFSUMB guidelines and recommendations on the clinical use of ultrasound elastography. Part 1: Basic principles and technology. U1traschall Med 2013; 34: 169-184.

20. Lupşor M, Badea R, Stefănescu H, et al. Analysis of histopathological changes that influence liver stiffness in chronic hepatitis C. Results from a cohort of 324 patients. J Gastrointestin Liver Dis 2008; 17: 155-163.

21. Saftoiu A, Vilmann P, Gorunescu F, et al. Endoscopic ultrasound elastography in the diagnosis of pancreatic cancer. Ann Gastroenterol 2010; 23: 200-201.

22. Cosgrove D, Piscaglia F, Bamber J, et al. EFSUMB guidelines and recommendations on the clinical use of ultrasound elastography. Part 2: Clinical applications. Ultraschall Med 2013; 34: 238-253.

23. Ferraioli G, Filice C, Castera L, et al. WFUMB guidelines and recommendations for clinical use of ultrasound elastography Part 3: Liver. Ultrasound Med Biol 2015; 41: 11611179.

24. Shaffer EA. Epidemiology and risk factors for gallstone disease: has the paradigm changed in the 21 st century? Curr Gastroenterol Rep 2005; 7: 132-140.

25. Kimura Y, Takada T, Kawarada Y, et al. Definitions, pathophysiology, and epidemiology of acute cholangitis and cholecystitis: Tokyo Guidelines. J Hepatobiliary Pancreat Surg 2007; 14: 15-26.

26. Rockey DC. Hepatobiliary infections. Curr Opin Gastroenterol 2001; 17: 257-261.

27. Kondo S, Isayama H, Akahane M, et al. Detection of common bile duct stones: comparison between endoscopic ultrasonography, magnetic resonance cholangiography, and helical-computed-tomographic cholangiography. Eur J Radiol 2005; 54: 271-275.
28. Baron RL, Stanley RJ, Lee JK, et al. A prospective comparison of the evaluation of biliary obstruction using computed tomography and ultrasonography. Radiology 1982; 145: 91-98.

29. Hong MJ, Kim SW, Kim HC, Yang DM. Comparison of the clinical characteristics and imaging findings of acute cholangitis with and without biliary dilatation. Br J Radiol 2012; 85: e1219-e1225.

30. Tantau M, Mercea V, Crisan D, et al. ERCP on a cohort of 2,986 patients with cholelitiasis: a 10-year experience of a single center. J Gastrointestin Liver Dis 2013; 22: 141-147.

31. Chapman R, Fevery J, Kalloo A, et al. Diagnosis and management of primary sclerosing cholangitis. Hepatology 2010; 51: 660-678.

32. Dave M, Elmunzer BJ, Dwamena BA, Higgins PD. Primary sclerosing cholangitis: meta-analysis of sclerosing cholangitis", section on ,Clinical management' diagnostic performance of MR cholangiopancreatography. Radiology 2010; 256: 387-396.

33. Kim HH, Hur YH, Koh YS, Cho CK, Kim JW. Intrahepatic biliary cystadenoma: Is there really an almost exclusively female predominance? World J Gastroenterol 2011; 17: 3073-3074.

34. Park SW, Song SY, Chung JB, et al. Endoscopic snare resection for tumors of the ampulla of Vater. Yonsei Med J 2000; 41: 213-218.

35. Xu HX, Chen LD. Villous adenoma of extrahepatic bile duct: contrast-enhanced sonography findings. J Clin Ultrasound 2008; 36: 39-41.

36. Gómez Iglesias S, Sendra León P, Mir Pallardo J, et al. Malignant tumors of the Vater's ampulla. Presentation of 28 cases and review of the literature. Rev Clin Esp 1994; 194: 9-12.

37. Laleman W, Verreth A, Topal B, et al. Endoscopic resection of ampullary lesions: a single-center 8-year retrospective cohort study of 91 patients with long-term follow-up. Surg Endosc 2013; 27: 3865-3876.

38. Lee SY, Jang KT, Lee KT, et al. Can endoscopic resection be applied for early stage ampulla of Vater cancer? Gastrointest Endosc 2006; 63: 783-788.

39. Cardinale V, Semeraro R, Torrice A, et al. Intra-hepatic and extra-hepatic cholangiocarcinoma: New insight into epidemiology and risk factors. World J Gastrointest Oncol 2010; 2: 407-416.

40. Bertuccio P, Bosetti C, Levi F, Decarli A, Negri E, La Vecchia C. A comparison of trends in mortality from primary liver cancer and intrahepatic cholangiocarcinoma in Europe. Ann Oncol 2013; 24: 1667-1674.

41. Ros PR, Buck JL, Goodman ZD, Ros AM, Olmsted WW. Intrahepatic cholangiocarcinoma: radiologic-pathologic correlation. Radiology 1988; 167: 689-693.

42. Wilson SR, Greenbaum LD, Goldberg BB. Contrast-enhanced ultrasound: what is the evidence and what are the obstacles? AJR Am J Roentgenol 2009; 193: 55-60.

43. Xu HX, Lu MD, Liu GJ, et al. Imaging of peripheral cholangiocarcinoma with low-mechanical index contrastenhanced sonography and SonoVue: initial experience. J Ultrasound Med 2006; 25: 23-33. 
44. Lin MX, Xu HX, Lu MD, et al. Diagnostic performance of contrast-enhanced ultrasound for complex cystic focal liver lesions: blinded reader study. Eur Radiol 2009; 19: 358-369.

45. Bolondi L. The appropriate allocation of CEUS in the diagnostic algorithm of liver lesions: a debated issue. Ultrasound Med Biol 2013; 39: 183-185.

46. Chen LD, Xu HX, Xie XY, et al. Enhancement patterns of intrahepatic cholangiocarcinoma: comparison between contrast-enhanced ultrasound and contrast-enhanced CT. Br J Radiol 2008; 81: 881-889.

47. Ahmetoglu A, Kosucu P, Kul S, et al. MDCT cholangiography with volume rendering for the assessment of patients with biliary obstruction. AJR Am J Roentgenol 2004; 183: $1327-1332$.

48. Kim MJ, Choi JY, Chung YE. Evaluation of biliary malignancies using multidetector-row computed tomography. J Comput Assist Tomogr 2010; 34: 496-505.

49. Vanderveen KA, Hussain HK. Magnetic resonance imaging of cholangiocarcinoma. Cancer Imaging 2004; 4: 104-115.

50. Corvera CU, Blumgart LH, Akhurst T, et al. 18F-fluorodeoxyglucose positron emission tomography influences management decisions in patients with biliary cancer. J Am Coll Surg 2008; 206: 57-65.

51. Xu HX, Chen LD, Xie XY, et al. Enhancement pattern of hilar cholangiocarcinoma: Contrast-enhanced ultrasound versus contrast-enhanced computed tomography. Eur J Radiol 2010; 75: 197-202.

52. Kim TK, Choi BI, Han JK, Jang HJ, Cho SG, Han MC. Peripheral cholangiocarcinoma of the liver: two-phase spiral CT findings. Radiology 1997; 204: 539-543.

53. Ajiki T, Fukumoto T, Ueno K, Okazaki T, Matsumoto I, Ku Y. Three-dimensional computed tomographic cholangiography as a novel diagnostic tool for evaluation of bile duct invasion of perihilar cholangiocarcinoma. Hepatogastroenterology 2013; 60: 1833-1838.

54. Fritscher-Ravens A, Broering DC, Knoefel WT, et al. EUSguided fine-needle aspiration of suspected hilar cholangiocarcinoma in potentially operable patients with negative brush cytology. Am J Gastroenterol 2004; 99: 45-51.

55. Manfredi R, Barbaro B, Masselli G, Vecchioli A, Marano P. Magnetic resonance imaging of cholangiocarcinoma. Semin Liver Dis 2004; 24: 155-164.

56. Park MS, Kim TK, Kim KW, et al. Differentiation of extrahepatic bile duct cholangiocarcinoma from benign stricture: findings at MRCP versus ERCP. Radiology 2004; 233: 234-240.

57. Choi BI, Lee JM. Neoplasms of the gallbladder and biliary tract. In: Textbook of gastrointestinal radiology. 3rd edition.
Gore RM, Levine MS (EDS.). Saunders Elsevier; Philadelphia, PA 2008: 1467-1489.

58. Inui K, Nakazawa S, Yoshino J, et al. Ultrasound probes for billiary lesions. Endoscopy 1998; 30(Suppl 1): A120-A123.

59. Marsh Rde W, Alonzo M, Bajaj S, et al. Comprehensive review of the diagnosis and treatment of biliary tract cancer 2012. Part I: diagnosis-clinical staging and pathology. J Surg Oncol 2012; 106: 332-338.

60. Dittrick GW, Mallat DB, Lamont JP. Management of ampullary lesions. Curr Treat Options Gastroenterol 2006; 9: 371-376.

61. Kim JH, Kim MJ, Chung JJ, Lee JT, Yoo HS, Lee JT. Differential diagnosis of periampullary carcinomas at MR imaging. Radiographics 2002; 22: 1335-1352.

62. Rösch T, Braig C, Gain T, et al. Staging of pancreatic and ampullary carcinoma by endoscopic ultrasonography. Comparison with conventional sonography, computed tomography, and angiography. Gastroenterology 1992; 102: 188-199.

63. Ito K, Fujita N, Noda Y, et al. Preoperative evaluation of ampullary neoplasm with EUS and transpapillary intraductal US: a prospective and histopathologically controlled study. Gastrointest Endosc 2007; 66: 740-747.

64. Baron RL, Tublin ME, Peterson MS. Imaging the spectrum of biliary tract disease. Radiol Clin North Am 2002; 40: 1325-1354.

65. Llop E, de Juan C, Seijo S, et al. Portal cholangiopathy: radiological classification and natural history. Gut 2011; 60: 853-860.

66. Dilawari JB, Chawla YK. Pseudosclerosing cholangitis in extrahepatic portalvenous obstruction. Gut 1992; 33: 272276.

67. Dhiman R1, Behera A, Chawla YK, Dilawari JB, Suri S. Portal hypertension biliopathy. Gut 2007; 56: 1001-1008.

68. Sandblom P. Hemobilia (Biliary Tract Hemorrhage): History, Pathology, Diagnosis, Treatment. 1st edition Springfield, Charles C. Thomas, 1972.

69. Curet P, Baumer R, Roche A, Grellet J, Mercadier M. Hepatic hemobilia of traumatic or iatrogenic origin: Recent advances in diagnosis and therapy, review of the literature 1976 to 1981 . World J Surg 1984; 8: 2-8.

70. Green MH, Duell RM, Johnson CD, Jamieson NV. Haemobilia. Br J Surg 2001; 88: 773-786.

71. Leoni S, Piscaglia F, Golfieri R, et al. The impact of vascular and nonvascular findings on the noninvasive diagnosis of small hepatocellular carcinoma based on the EASL and AASLD criteria. Am J Gastroenterol 2010; 105: 599-609. 